\title{
Acquired symptomatic external punctal stenosis: A tertiary referral center study
}

\author{
Mona Nassief ${ }^{1}$, Omar Kh M Alduwailah ${ }^{2}$ and Nancy M. Lotfy ${ }^{3}$ \\ ${ }^{1}$ Department of Ophthalmology, Faculty of Medicine, Tanta University, Tanta, Egypt \\ ${ }^{2}$ Department of Ophthalmology, Farwaniya hospital, Farwaniya, Kuwait \\ ${ }^{3}$ Department of Ophthalmology, Faculty of Medicine, Cairo University, Cairo, Egypt
}

\begin{abstract}
Background: To report the prevalence of acquired symptomatic external punctal stenosis (EPS) in a tertiary referral center in Kuwait, and to study the associated risk factors, etiologies, and management outcomes. Methods: This prospective interventional hospital-based study was performed at the Farwaniya governmental hospital in Kuwait between November 2018 and November 2019. All patients were referred to the oculoplastic clinic with symptomatic epiphora, age $>18$ years, epiphora Munk score $\geq 0$, tear film meniscus $\geq 2 \mathrm{~mm}$, and punctum with grade $\leq 2$ (smaller than normal size, but recognizable) were included. Exclusion criteria were congenital causes of epiphora, previous eyelid surgeries, and traumatic or neoplastic causes of punctal or canalicular obstruction.

Results: A total of 418 patients with symptomatic epiphora, who were referred to our oculoplastic clinic, were enrolled in our study. The prevalence of EPS was 70.3\%. Examination of the puncta revealed different shapes, including tangential, pinpoint, elevated, slit-shaped, horse shoe-shaped, and membranous puncta, with the tangential type accounting for the greatest proportion (65.3\%). Bilateral punctal stenosis was observed in 206 patients $(70.1 \%)$ and unilateral involvement in 88 patients $(29.9 \%)$. Dilatation and syringing were performed for all puncta upon diagnosis, and repeated dilatation was performed for 225 puncta $(32.7 \%)$. Placement of a Mini-Monoka tube was performed in 11 puncta (1.6\%).

Conclusion: EPS was a common cause of symptomatic epiphora in our study. Aging and female sex were identified as common risk factors. Most etiological factors of EPS were associated with a pathophysiological inflammatory mechanism. For exact estimates of its prevalence, a population-based study is necessary in future.
\end{abstract}

\section{KEY WORDS}

punctal stenosis, dry eye, chronic blepharitis, mini monoka, Kuwait, prevalence, epiphora

\section{INTRODUCTION}

External punctal stenosis (EPS) in both congenital and acquired forms is a common disorder that affects the punctum of the eye, and it is among the common causes of symptomatic epiphora [1]. The punctum is a small aperture on the slight tissue elevation (lacrimal papilla) at the junction between the lacrimal and ciliary parts of the eyelid margin, in both eyelids. Normally, the puncta are turned toward the globe and are visible only if the eyelid margin is everted slightly. Each punctum leads into a tube called the lacrimal canaliculus [2].

Anatomically, punctal stenosis is defined as a narrowing or occlusion of the external opening of the lacrimal canaliculus, which is located in the nasal part of the lid margin. According to the grading system defined by Kashkouli et al., the external punctal opening size can be classified into six categories: grade 0 (punctal atresia),

Correspondence: Nancy M. Lotfy MD, Department of Ophthalmology, Faculty of Medicine, Cairo University, Cairo, Egypt. Email: nancymaher@kasralainy. edu.eg. ORCID iD: https://orcid.org/0000-0002-9159-0020

How to cite this article: Nassief M, Alduwailah OKM, Lotfy NM. Acquired symptomatic external punctal stenosis: A tertiary referral center study. Med Hypothesis Discov Innov Ophthalmol. 2021 Spring; 10(1): 18-23. https://doi.org/10.51329/mehdiophthal1417

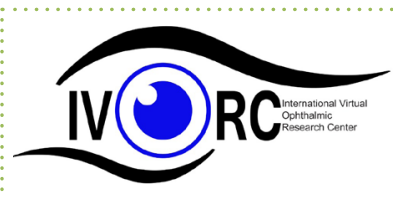

Copyright (C) Author(s). This is an open-access article distributed under the terms of the Creative Commons Attribution-NonCommercial 4.0 International License (http://creativecommons.org/licenses/by-nc/4.0/) which permits copy and redistribute the material just in noncommercial usages, provided the original work is properly cited. (c) (i) (5) 
grade 1 (papilla is covered by a membrane or fibrosis which is difficult to recognize), grade 2 (smaller than normal size, yet recognizable), grade 3 (normal size), grade 4 (small slit $<2 \mathrm{~mm}$ ), and grade 5 (large slit $\geq 2 \mathrm{~mm}$ in size). All cases with acquired EPS are grade 1 or 2 on this grading system. EPS can involve puncta with different acquired shapes, including elevated, tangential, membranous, pinpoint, horse shoe-shaped, and slit-shaped puncta [3].

The prevalence of EPS increases with age due to involutional and fibrotic changes. Causes of acquired EPS include inflammation, such as eyelid infections, blepharitis, dry eye, graft-versus-host disease, ocular cicatricial pemphigoid, eyelid malposition, infections with pathogens such as Chlamydia trachomatis, herpes virus, human papilloma virus, and actinomyces species. Topical medications, such as timolol, latanoprost, betaxolol, pilocarpine, adrenaline, chloramphenicol, prednisolone acetate, phenylephrine hydrochloride, mitomycin-C, artificial tears, naphazoline, and tobramycin, as well as systemic medications, such as 5-fluorouracil can cause acquired EPS. Moreover, acquired EPS can also be related to neoplasms or trauma, or the condition can be idiopathic [4-6].

The prevalence of EPS ranges from $17.3 \%$ to $54.3 \%[1,7]$. However, the epidemiology of the disease in the Middle East has not been extensively studied, and to the best of our knowledge, the prevalence of symptomatic EPS in Kuwait has not been reported. Here, we investigate the prevalence, underlying etiologies, associated risk factors, and management outcomes of EPS in Kuwait.

\section{METHODS}

This prospective interventional single-center study was performed at the Farwaniya governmental hospital in Kuwait between November 2018 and November 2019. The study protocol was reviewed and approved by the regional medical ethics committee and was performed in accordance with the tenets of the Declaration of Helsinki. Written informed consent was obtained from all patients.

We included patients who were referred to the oculoplastic sub-specialty clinic who presented with symptomatic epiphora, were $>18$ years of age, had an epiphora Munk score $\geq 0$ (Table 1), tear film meniscus $\geq 2 \mathrm{~mm}$, and a punctal opening with grade $\leq 2$ on the Kashkouli grading system.

Patients with congenital causes of epiphora, previous eyelid surgeries, traumatic or neoplastic causes of punctal or canalicular obstruction, or any other causes of lacrimal passage obstruction, such as canalicular, common canalicular, or nasolacrimal duct obstruction were excluded from the study.

All patients' demographic data, a detailed history of eye and systemic diseases, topical or systemic medications used, and smoking history were recorded. Thereafter, all patients underwent a comprehensive examination of the ocular surface and anterior and posterior segments using slit-lamp biomicroscopy (Topcon, Livermore, CA, USA). Punctum examination and grading were performed using slit lamp biomicroscopy. Using the Kashkouli et al. grading system [3], only eyes with grade 1 (papilla is covered by fibrosis or a membrane and is difficult to identify), and grade 2 (smaller than normal size but identifiable) were included in our study.

A comprehensive examination of the eyelids, conjunctiva, and cornea was performed. Examination of meibomian gland size, number, plugging of orifices, type of secretions, and expression of contents were performed to detect signs of meibomian gland dysfunction (MGD). The ocular surface was evaluated by measuring the tear film breakup time (TBUT), and performing the Schirmer test and fluorescein clearance test. TBUT [9] was determined by applying a drop of $1 \%$ sodium fluorescein in the lower fornix. The patient was instructed to blink, and the tear film was then examined using a slit lamp and a cobalt blue filter. The time between the last blink and the appearance of the first dry spot was documented. A TBUT value of $<10 s$ was considered to be abnormal. The Schirmer test [10] was performed after application of a drop of $0.4 \%$ oxybuprocaine hydrochloride in the lower conjunctival sac, and then a standard Schirmer strip (Tear Flo, HUB Pharmaceuticals, Rancho Cucamonga, CA, USA) was placed in the lower temporal fornix for $5 \mathrm{~min}$. Wetting of filter paper $\leq 5 \mathrm{~mm}$ was considered to represent a positive Schirmer test. The fluorescein clearance test [11] was performed by applying one drop of $2 \%$ sterile fluorescein solution into the conjunctival fornices of both eyes, after which the tear film was examined using the cobalt blue filter of the slit lamp. The persistence of a significant amount of dye after 5 min denoted an obstruction.

Table 1. Munk scoring for epiphora [8]

\begin{tabular}{|l|l|}
\hline Score & Definition \\
\hline $\mathbf{0}$ & No epiphora \\
\hline $\mathbf{1}$ & Occasional epiphora that requires drying $<2$-time a day. \\
\hline $\mathbf{2}$ & Epiphora that requires dabbing $5-10$ times a day. \\
\hline $\mathbf{3}$ & Epiphora that requires dabbing $>10$ times a day. \\
\hline $\mathbf{4}$ & Epiphora that requires dabbing daily or constant tearing. \\
\hline
\end{tabular}


In terms of management, all cases with EPS underwent dilatation and syringing, followed by treatment with preservative-free anti-inflammatory eye drops twice daily, and tear substitute eye drops five times a day. Patients were followed up at 2-week, 1, 3, and 6 months post-dilatation. Outcomes were evaluated objectively using the fluorescein clearance test, measuring the punctal size, and subjectively using the Munk score [8].

During the follow-up period, any documented recurrence of symptoms and signs was treated by re-dilatation of the punctum, with subsequent follow-up. Failure of the second dilatation procedure was treated by insertion of a Mini Monoka tube (Fayet and Bernard, FCI Ophthalmics, Paris, France). The associated risk factors, such as ectropion and MGD, were treated according to the standard treatment approach. All examinations and management were performed by a single experienced oculoplastic ophthalmology consultant, using the same instrumentation.

Statistical analysis was performed using IBM SPSS Statistics for Windows, version 26.0, (IBM Corp., Armonk, NY, USA). Qualitative variables are summarized as frequencies (number and percentage). Pearson's chi-square test for independence, Fisher's exact test, or the Fisher-Freeman-Halton exact test were used to examine the association between two categorical variables, as appropriate. A P $<0.05$ was interpreted as statistically significant.

\section{RESULTS}

A total of 418 patients with symptomatic epiphora, who were referred to the oculoplastic clinic, were enrolled in our study. The prevalence of EPS was 70.3\% (294 patients). Among the patients, 137 (46.6\%) were men and 157 (53.4\%) were women. The mean \pm standard deviation (SD) of age was $54.7 \pm 12.6$ years. Table 2 shows the prevalence of different predisposing factors and different shapes of puncta in the study patients. Many predisposing factors were associated with EPS, and in most cases, more than one factor was present. Examination of the puncta revealed different shapes, including tangential, pinpoint, elevated, slit-shaped, horse shoe-shaped, and membranous puncta, with the tangential type accounting for the greatest proportion (65.3\%) (Table 2).

Based on Kashkouli et al.s grading system [3], 121 (17.6\%) puncta were grade 1 and 567 (82.4\%) were grade 2. Among patients with EPS, punctal stenosis was bilateral in 206 patients (70.1\%) and unilateral in 88 patients (29.9\%). Regarding the number of stenosed puncta, only one stenosed punctum was seen in 61 patients $(20.7 \%)$, two stenosed puncta occurred in 103 patients $(35,0 \%)$, three stenosed puncta in 99 patients $(33.7 \%)$, and four stenosed puncta in 31 patients $(10.5 \%)$

Table 2. Frequency of different predisposing factors and different shapes of puncta for symptomatic external punctal stenosis

\begin{tabular}{|l|l|l|l|}
\hline Predisposing factor & n $(\%)$ & Tangential & $449(65.3)$ \\
\hline Post-viral infection & $254(86.4)$ & Pin point & $241(35.0)$ \\
\hline Topical eye drops & $242(82.3)$ & Elevated & $239(34.7)$ \\
\hline Dry eye & $241(82.0)$ & Slit-shaped & $207(30.1)$ \\
\hline Blepharitis & $212(72.1)$ & Horse shoe-shaped & $132(19.2)$ \\
\hline Use of cosmetics & $131(44.6)$ & Membranous & $108(15.7)$ \\
\hline Contact lens wear & $50(17.0)$ & & \\
\hline Lower lid laxity & $\begin{array}{l}\text { Mild 32 }(10.9) \\
\text { Moderate 3(1.0) } \\
\text { Severe } 1(0.3)\end{array}$ & & \\
\hline History of malignancy & $4(1.4)$ & & \\
\hline
\end{tabular}

Abbreviations: n, number; \%, percentage.

Table 3. Follow up of patients symptomatic external punctal stenosis based on Munk scoring $[8]$ for epiphora

\begin{tabular}{|c|c|c|c|c|c|c|c|}
\hline Examination & Grade 0, & Grade 1 & Grade 2 & Grade 3 & Grade 4 & Total & P-value \\
\hline Pre-operative, n (\%) & $0(0.0)$ & $0(0.0)$ & $102(20.4)$ & $297(59.4)$ & $101(20.2)$ & $500(100.0)$ & \\
\hline 2-week post-operative, n (\%) & $385(77.0)$ & $76(15.2)$ & $30(6.0)$ & $5(1.0)$ & $4(0.8)$ & $500(100.0)$ & $P_{1}<0.001$ \\
\hline 1-month post-operative, n (\%) & $384(77.7)$ & $72(14.6)$ & $32(6.5)$ & $4(0.8)$ & $2(0.4)$ & $494(100.0)$ & $\begin{array}{l}\boldsymbol{P}_{\mathbf{1}}<\mathbf{0 . 0 0 1} \\
P_{2}=0.938\end{array}$ \\
\hline 3-month post-operative, n (\%) & $379(78.3)$ & $76(15.7)$ & $29(6.0)$ & $0(0.0)$ & $0(0.0)$ & $484(100.0)$ & $\begin{array}{l}P_{1}<\mathbf{0 . 0 0 1} \\
P_{3}=0.212\end{array}$ \\
\hline 6-month post-operative, n (\%) & $364(76.5)$ & $82(17.2)$ & $30(6.3)$ & $0(0.0)$ & $0(0.0)$ & $476(100.0)$ & $\begin{array}{l}P_{1}<\mathbf{0 . 0 0 1} \\
P_{4}=0.786\end{array}$ \\
\hline
\end{tabular}

Abbreviations: n, number; \%, percentage. Note: $\boldsymbol{P}_{1}$, comparison with preoperative score; $\boldsymbol{P}_{2}$, comparison with 2 -weeks score; $\boldsymbol{P}_{3}$, comparison with first-month score; $\boldsymbol{P}_{4}$, comparison with third-month score (Pearson's Chi square test $/$ Fisher-Freeman-Halton exact test); $\boldsymbol{P}$-value $<0.05$ is shown in bold. 
Table 4. Follow up of patients with symptomatic external punctal stenosis based on based on the Kashkouli [3] grading system (punctal size)

\begin{tabular}{|c|c|c|c|c|c|c|}
\hline Examination & Grade 1 & Grade 2 & Grade 3 & Grade 4 & Total & P-value \\
\hline Pre-operative, n (\%) & $121(17.6)$ & $567(82.4)$ & $0(0.0)$ & $0(0.0)$ & $688(100.0)$ & \\
\hline 2-week post-operative, n (\%) & $9(1.3)$ & $278(40.4)$ & $251(36.5)$ & $150(21.8)$ & $688(100.0)$ & $P_{1}<0.001$ \\
\hline 1-month post-operative, n (\%) & $1(0.1)$ & $277(41.3)$ & $246(36.7)$ & $146(21.8)$ & $670(100.0)$ & $\begin{array}{l}\boldsymbol{P}_{\mathbf{1}}<\mathbf{0 . 0 0 1} \\
P_{2}=0.099\end{array}$ \\
\hline 3-month post-operative, n (\%) & $1(0.2)$ & $222(36.8)$ & $241(40.0)$ & $139(23.1)$ & $603(100.0)$ & $\begin{array}{l}\boldsymbol{P}_{\mathbf{1}}<\mathbf{0 . 0 0 1} \\
P_{3}=0.375\end{array}$ \\
\hline 6-month post-operative, n (\%) & $0(0.0)$ & $217(37.4)$ & $231(39.8)$ & $132(22.8)$ & $580(100.0)$ & $\begin{array}{l}\boldsymbol{P}_{\mathbf{1}}<\mathbf{0 . 0 0 1} \\
P_{4}=0.990\end{array}$ \\
\hline
\end{tabular}

Abbreviations: n, number; \%, percentage. Note: $\boldsymbol{P}_{1}$, comparison with preoperative score; $\boldsymbol{P}_{2}$, comparison with 2-weeks score; $\boldsymbol{P}_{3}$, comparison with first-month score; $\boldsymbol{P}_{4}$, comparison with third-month score (Pearson's Chi square test/Fisher-Freeman-Halton exact test); $\boldsymbol{P}$-value $<0.05$ is shown in bold.

Dilatation and syringing were performed for all puncta upon diagnosis, and repeated dilatation was performed in 225 puncta (32.7\%). Placement of a Mini Monoka tube was performed in 11 (1.6\%) patients. To manage EPS associated ectropion, full-thickness wedge resection with lower blepharoplasty was performed in two patients (0.3\%). Lateral canthoplasty was performed in two patients $(0.3 \%)$.

Tables 3 and 4 show the follow-up results of patients at 2-weeks and at 1 month, 3 months, and 6 months postdilatation, based on the epiphora scale and grades of punctal occlusion, respectively.

\section{DISCUSSION}

In the current study EPS was a common cause of symptomatic epiphora. Aging and female sex were identified as common risk factors. Most etiological factors of EPS were associated with a pathophysiological inflammatory mechanism. The prevalence of EPS was $70.3 \%$ (294 patients).

The prevalence of EPS has been estimated to range between $17.3 \%$ and $54.3 \%[1,7]$. A recent study conducted in Turkey revealed a prevalence of EPS among the older population of 63.3\% [12]. However, these prevalence estimates represented all cases of EPS, irrespective of whether they were symptomatic or asymptomatic. In this hospital-based study, we report the prevalence of only symptomatic EPS patients who presented with epiphora. We found a prevalence of $70.3 \%$, which is higher than that reported in previous studies.

Sixty-seven percent of our cases were above 50 years of age, with a mean \pm SD age of $54.7 \pm 12.6$ years. Old age has been described as a causative factor for EPS in many studies, and involutional and fibrotic changes associated with aging are thought to be the causative mechanisms $[1,3,7,12]$. Although we reported a high prevalence among older age groups, our prevalence estimates differ from those of other studies, which reported prevalence ratios ranging from only $1 \%$ in patients aged $40-49$ years to $>50 \%$ in older patients $[1,3,13]$. This discrepancy in results could be explained by the emergence of other risk factors for EPS that could affect younger age groups, such as post-viral infection, dry eye, frequent use of topical eye drops, use of contact lenses, and use of eye cosmetics.

The higher prevalence rate in women (53.4\%) in our study is consistent with some previously reported studies. The authors of those studies attributed this sex-related bias to hormonal changes either in post-menopausal [3] or perimenopausal years [14], we found a greater proportion of affected females in the older age groups. However, other authors have not reported any sex-related differences $[7,12]$.

The overall high prevalence found in our study could be attributed to the multifactorial etiology of the disease. In our study, the most common associated risk factors were post-viral infection (86.4\%), topical eye drop use $(82.3 \%)$, dry eye (82\%), and blepharitis (72.1\%). Most of the etiologies of EPS are associated with a pathophysiological inflammatory mechanism [15]. Viral conjunctivitis is considered the most common etiology of infectious conjunctivitis [16-19]. In our study, we found a high prevalence of EPS that occurred post-viral conjunctivitis $(86.4 \%)$. A study conducted by Yulish et al. concluded that viral conjunctivitis and associated chronic inflammation could be a cause of EPS [20].

In the present study, chronic blepharitis was diagnosed in $72.1 \%$ of cases. The association between chronic blepharitis and EPS has been documented in many studies in the literature. The authors suggested that blepharitisassociated chronic inflammation and fibrosis can lead to the formation of inflammatory membranes, overgrowth of the conjunctival epithelium, and keratinization around the punctum, leading to stenosis and obstruction [1, $3,12]$.

One of the most common associated risk factors in our study was the frequent use of topical eye drops 
(82.3\%). Obstruction of the lacrimal canalicular system, including the puncta, could occur after eye drop installation, and a significant association has been found between glaucoma drop use and EPS [12]. It has been suggested that fibrotic reactions might involve the epithelial and sub-epithelial tissues of the lacrimal canaliculi and puncta, leading to progressive stenosis, followed by obstruction. Preservatives used in topical eye drops may be responsible for these clinical changes $[6,12,15,21]$.

Dry eye is a chronic disease with a reported worldwide prevalence that varies from $5 \%$ to $50 \%$. Risk factors include aging, female sex, MGD, computer use, contact lens wear, and environmental factors, such as low humidity, high temperature, wind, and pollution [22]. Dry eye was found in $82 \%$ of EPS cases in our study. This high prevalence of dry eye could be explained by the high prevalence of the predisposing diseases, frequent use of tear replacement eye drops, and associated chronic blepharitis.

This study reports four cases (1.4\%) of EPS that occurred after chemotherapy treatment of malignant tumors (three cases of breast cancer and one case of colon cancer). EPS after chemotherapy could be due to local or systemic effects. Local effects could result from drug secretion in the tear film, which can lead to punctal stenosis as a result of direct contact with the drug. Systemic effects could be due to fibrosis of the mucous membrane of the puncta secondary to systemic drug effects [5].

In our study, we included symptomatic cases with grade 1 (papilla is covered by a membrane or fibrosis and is hard to identify), and grade 2 (smaller than normal size but identifiable) EPS. Grade 0 (punctal atresia) was excluded, as it requires surgical creation of a papilla, which was outside of the scope of our study [3]. We found $121(17.6 \%)$ patients with grade 1 and 567 (82.4\%) with grade 2. Different shapes of puncta were found, with the tangential type accounting for the greatest proportion (65.3\%).

The management of cases included dilatation and syringing followed by treatment with preservative-free antiinflammatory eye drops, twice daily, and tear substitutes eye drops, five times daily. Patients were followed-up at 2 -weeks, and at 1, 3, and 6 months post-dilatation. Re-dilatation was required in 225 puncta (32.7\%) cases, to which 11 puncta did not respond and were finally treated by insertion of a Mini Monoka tube. The Mini Monoka mono-canalicular stent is a silicone tube used in the management of EPS [23]. Treatment of associated ectropion was performed by full-thickness wedge resection with lower blepharoplasty in two puncta (0.3\%), and lateral canthoplasty in two puncta $(0.3 \%)$.

This study had some limitations. This was a hospital-based study; however, a population-based cohort study would offer a better understanding of the nature of the disease. Another limitation is that all examinations, classifications, and management were performed by a single ophthalmologist, which hinders reproducibility assessments. We used a clinical sample instead of a population-based sample for prevalence estimates, which is considered another limitation of our study. Therefore, our results may not represent the actual prevalence of EPSs. However, to the best of our knowledge, no previous study had reported the prevalence of symptomatic EPS in Kuwait. Moreover, we investigated the underlying etiologies, associated risk factors, and management outcomes of EPS in this population. For exact prevalence estimates, we would recommend a population-based study in future. Furthermore, future studies are warranted to investigate the etiologies of other lacrimal drainage system disorders.

\section{CONCLUSIONS}

In this study, EPS was a common cause of symptomatic epiphora. We found a high prevalence of this disease. Aging and female sex were risk factors, although many other risk factors, such as vitamin D deficiency, post-viral infection, topical eye drop use, and dry eye, were identified. Most of the etiologies of EPS are associated with a pathophysiologic inflammatory mechanism.

\section{ETHICAL DECLARATIONS}

Ethical approval: The study protocol was reviewed and approved by the regional medical ethics committee and was in accordance with the tenets of the Declaration of Helsinki. Informed written consent was obtained from all patients.

Conflict of interests: None.

\section{FUNDING}

\section{None.}




\section{ACIKNOWLED GMENT}

We would like to express our gratitude to all patients, professors, and colleagues; this work could not have been accomplished without their support.

\section{REFERENCES}

1. Bukhari A. Prevalence of punctal stenosis among ophthalmology patients. Middle East Afr J Ophthalmol. 2009;16(2):85-7. doi: 10.4103/0974-9233.53867 pmid: 20142967

2. Remington LA. Ocular adenexa and lacrimal system. clinical anatomy and physiology of the visual system2012.p. 159-81.

3. Kashkouli MB, Beigi B, Murthy R, Astbury N. Acquired external punctal stenosis: etiology and associated findings. Am J Ophthalmol. 2003;136(6):1079-84. doi: 10.1016/s0002-9394(03)00664-0 pmid: 14644218

4. Ahmed RA, Kamal AM, Zayed MA. Evaluation of planned bicanalicular nasolacrimal tube retention in treating punctal stenosis or occlusion secondary to trachoma. Orbit. 2017;36(4):208-14. doi: 10.1080/01676830.2017.1337160 pmid: 28641033

5. Esmaeli B, Valero V, Ahmadi MA, Booser D. Canalicular stenosis secondary to docetaxel (taxotere): a newly recognized side effect. Ophthalmology. 2001;108(5):994-5. doi: 10.1016/s0161-6420(00)00640-0 pmid: 11320034

6. Soiberman U, Kakizaki H, Selva D, Leibovitch I. Punctal stenosis: definition, diagnosis, and treatment. Clin Ophthalmol. 2012;6:1011-8. doi: 10.2147 /opth.s31904 pmid: 22848141

7. Viso E, Rodriguez-Ares MT, Gude F. Prevalence and associations of external punctal stenosis in a general population in Spain. Cornea. 2012;31(11):1240-5. doi: 10.1097/ICO.0b013e31823f8eca pmid: 22367046

8. Girard B, Piaton JM, Keller P, Nguyen TH. Botulinum neurotoxin A injection for the treatment of epiphora with patent lacrymal ducts. J Fr Ophtalmol. 2018;41(4):343-9. doi: 10.1016/j.jfo.2017.11.010 pmid: 29681465

9. Wang MTM, Craig JP. Comparative Evaluation of Clinical Methods of Tear Film Stability Assessment: A Randomized Crossover Trial. JAMA Ophthalmol. 2018;136(3):291-4. doi: 10.1001/jamaophthalmol.2017.6489 pmid: 29392287

10. Li N, Deng XG, He MF. Comparison of the Schirmer I test with and without topical anesthesia for diagnosing dry eye. Int J Ophthalmol. 2012;5(4):478-81. doi: 10.3980/j.issn.2222-3959.2012.04.14 pmid: 22937509

11. Garaszczuk IK, Montes Mico R, Iskander DR, Expósito AC. The tear turnover and tear clearance tests - a review. Expert Rev Med Devices. 2018;15(3):219-29. doi: 10.1080/17434440.2018.1435271 pmid: 29384019

12. Ulusoy MO, Atakan M, Kivanc SA. Prevalence and associated factors of external punctal stenosis among elderly patients in Turkey. Arq Bras Oftalmol. 2017;80(5):296-9. doi: 10.5935/0004-2749.20170072 pmid: 29160539

13. Bukhari A. Etiology of tearing in patients seen in an oculoplastic clinic in Saudi Arabia. Middle East Afr J Ophthalmol. 2013;20(3):198200. doi: 10.4103/0974-9233.114790 pmid: 24014980

14. Kornhauser T, Segal A, Walter E, Lifshitz T, Hartstein M, Tsumi E. Idiopathic edematous punctal stenosis with chronic epiphora: preponderance in young women. Int Ophthalmol. 2019;39(9):1981-6. doi: 10.1007/s10792-018-1031-y pmid: 30269311

15. Port AD, Chen YT, Lelli GJ, Jr. Histopathologic changes in punctal stenosis. Ophthalmic Plast Reconstr Surg. 2013;29(3):201-4. doi: 10.1097/IOP.0b013e31828a92b0 pmid: 23552606

16. Uchio E, Takeuchi S, Itoh N, Matsuura N, Ohno S, Aoki K. Clinical and epidemiological features of acute follicular conjunctivitis with special reference to that caused by herpes simplex virus type 1. Br J Ophthalmol. 2000;84(9):968-72. doi: 10.1136/bjo.84.9.968 pmid: 10966946

17. Solano D, Fu L, Czyz CN. Viral Conjunctivitis. 2021 Feb 3. In: StatPearls [Internet]. Treasure Island (FL): StatPearls Publishing; 2021 Jan-. pmid: 29262100. Bookshelf ID: NBK470271

18. Azari AA, Arabi A. Conjunctivitis: A Systematic Review. J Ophthalmic Vis Res. 2020;15(3):372-95. doi: 10.18502/jovr.v15i3.7456 pmid: 32864068

19. Johari Moghadam MM, Mohamad Yari M, Azizi Jalilian F, Amini R, Bazzazi N. Epidemiology and molecular diagnosis of acute conjunctivitis in patients attending Hamadan, west Iran ophthalmology clinics 2016-2017. Clin Optom (Auckl). 2019;11:105-11. doi: 10.2147/ opto.s217722 pmid: 31686938

20. Yulish M, Pikkel J. Presumed Virus-Induced Punctal Occlusion. Adv Med. 2014;2014:809851. doi: 10.1155/2014/809851 pmid: 26556428

21. McNab AA. Lacrimal canalicular obstruction associated with topical ocular medication. Aust N Z J Ophthalmol. 1998;26(3):219-23. doi: 10.1111/j.1442-9071.1998.tb01315.x pmid: 9717753

22. Stapleton F, Alves M, Bunya VY, Jalbert I, Lekhanont K, Malet F, et al. TFOS DEWS II Epidemiology Report. Ocul Surf. 2017;15(3):33465. doi: $10.1016 /$ j.jtos.2017.05.003 pmid: 28736337

23. Kashkouli MB, Beigi B, Astbury N. Acquired external punctal stenosis: surgical management and long-term follow-up. Orbit. 2005;24(2):73-8. doi: 10.1080/01676830490916055 pmid: 16191791 